\title{
Desmoid Type Fibromatosis of the Breast: Ten-Year Institutional Results of Imaging, Histopathology, and Surgery
}

\author{
Jörn Lorenzen ${ }^{a}$ Miriam Cramer $^{\mathrm{a}}$ Nina Buck $^{\mathrm{a}}$ Kay Friedrichs ${ }^{\mathrm{b}}$ \\ Kirsten Graubner ${ }^{c}$ Clara Sonja Lühr ${ }^{d}$ Christoph Lindner ${ }^{c} \quad$ Axel Niendorf ${ }^{d}$ \\ ${ }^{a}$ Radiologische Allianz, Hamburg, Germany; ${ }^{b}$ Mammazentrum Hamburg, Hamburg, Germany; ${ }^{\mathrm{c} D e p a r t m e n t}$ \\ of Gynecology and Obstetrics, Agaplesion Diakonieklinikum Hamburg, Hamburg, Germany; ${ }^{d}$ MVZ Pathologie \\ Hamburg-West, Hamburg, Germany
}

\section{Keywords}

Desmoid type fibromatosis · Follow-up · Imaging ·

Immunohistochemistry $\cdot$ Recurrence $\cdot$ Resection margins

\begin{abstract}
Background: Desmoid type fibromatoses has proven to be a diagnostic and therapeutic challenge, as they often appear primarily as a carcinoma of the breast with a high recurrence risk. Patients: A digital archive search was performed for the period from 2009 to the end of 2018. Inclusion criteria consisted of histological examination of at least the surgical specimen in the reference pathology department and at least a second opinion diagnosis in the reference radiology department. Results: A total of 14 women and 1 man underwent surgery on desmoid type fibromatosis of the breast. The average patient age was 49 years (range: $22-72$ years). The mean tumor size was $2.2 \mathrm{~cm}$ (range: $0.8-4.2 \mathrm{~cm}$ ). The tumor was detectable in mammography in 12 out of 13 patients and in all 15 patients in sonography. MRI was performed preoperatively in 6 patients; in all of the patients, the tumor was visualized with inhomogeneous contrast enhancement. In the imaging procedures, all desmoid type fibromatoses were classified as suspicious. Performing the core biopsy, preoperative histology confirmed desmoid fibromatosis in 12 out of 15 patients. Nuclear stain for ß-
\end{abstract}

catenin was positive in 7 out of 10 patients. Negative staining was found for AE1/A3 in 10 out of 10 and CD34 in 12 out of 12 patients. In all of the patients, a single-stage operation without the detection of border-forming tumor margins was performed. The follow-up interval ranged from 16 to 96 months (mean: 44.86 months, median: 43 months). In this follow-up period, no patient was diagnosed with desmoid tumor recurrence. Conclusion: In imaging, desmoid type fibromatosis of the breast has typical malignancy-related criteria. Extensive preoperative diagnostics enable the planning of complete primary excision of the lesion and reduce the recurrence risk.

(-) 2020 The Author(s) Published by S. Karger AG, Basel

\section{Introduction}

Desmoid type fibromatosis is a locally aggressive growing tumor entity that does not metastasize. The main locations include the mesentery, the abdominal wall, and the extremities $[1,2]$. Here, they form $4 \%$ of the extraabdominal manifestations of this tumor entity and only $0.2 \%$ of all breast tumors [3-6]. Due to their rarity, they have proven to be a diagnostic and therapeutic challenge, as they often appear primarily as a carcinoma of the breast and are prone to recurrence [5-7].
() 2020 The Author(s)

Published by S. Karger AG, Basel

This is an Open Access article licensed under the Creative Common Attribution-NonCommercial-4.0 International License (CC BY-NC) (http://www.karger.com/Services/OpenAccessLicense), applicable to the online version of the article only. Usage and distribution for commercial purposes requires written permission. 
According to the current consensus from existing research, e.g., of the Desmoid Tumor Working Group and ESMO, active primary surgical therapy is no longer the method of choice for asymptomatic patients $[8,9]$. In the more recent literature on desmoid type fibromatosis of the breast an active surveillance approach showed progression-free courses or remissions in up to $88 \%$ of patients $[10,11]$.

However, surgical therapy remains a valuable therapeutic option, especially in symptomatic and progressive desmoid type fibromatosis or impaired quality of life [8, $9,12,13]$.

Therefore, imaging and a histopathological diagnosis are of great importance for therapeutic planning und follow-up results $[4,12,13]$. Large case numbers of breast desmoid type fibromatosis (BDF) are rare and have been published in retrospective evaluations focusing on clinical outcomes and therapy $[5,14,15]$. Current systematic evaluations of pre- and postoperative histology, taking into account immunohistochemistry, with evaluations of imaging findings and the clinical course, are predominantly single case reports [16-18].

In this retrospective study of primarily surgically treated patients with desmoid type fibromatosis, we report the clinical, diagnostic, pathological, and surgical results of this rare breast tumor manifestation and discuss the results of our institutional experience in comparison to those in the existing literature.

\section{Materials and Methods}

Based on a digital archive search in the reference pathology and reference radiology departments for the period from 2009 to the end of 2018, a total of 15 patients were identified under the heading "desmoid" or "fibromatosis." The prerequisite was a postoperative histological examination of the surgical specimen in the reference pathology department, at least a second diagnostic evaluation in the reference radiology department, and follow-up after surgery for a period of at least 12 months. Breast surgery was performed at 2 certified breast centers. The radiology and pathology departments were the cooperation partners of these breast centers. The pathological findings were independently reported twice. Details of the resection margins of the tumor to healthy tissue were available for all of the patients. In addition, the patient had to have the breast center surgical report and the final medical report. The preoperative radiological findings, including digital imaging findings, of all of the patients were available and they were re-evaluated in the current study. All of the patients underwent preoperative imaging or a secondary evaluation of external imaging in the participating radiology department. All of the patients underwent an additional clinical examination as part of the second radiographic assessment, and the sonographic examination was repeated. An additional mammography or magnetic resonance imaging (MRI) of the breast was performed when necessary. As part of the follow-up, all of the patients underwent clinical and imaging examinations.

The following data were evaluated: age, pre- and postoperative histological diagnosis, tumor size, distance of the resection margins, breast surgeries to date, follow-up, and clinical and imaging findings.

\section{Results}

\section{Patients}

A total of 14 women and 1 man with the diagnosis of a desmoid type fibromatosis of the breast were operated on during this period. The average patient age was 49 years (range: $22-72$ years). The mean tumor size was 2.2 $\mathrm{cm}$ (range: $0.8-4.2 \mathrm{~cm}$, median: $1.5 \mathrm{~cm}$ ). The tumor size was determined on whole-mount histological sections.

Two patients underwent simultaneous contralateral breast cancer surgery. According to the anamnesis of 2 other patients, 1 with a mammary carcinoma underwent breast-conserving surgery 4 or 9 years prior to the development of BDF in the ipsilateral breast, and 1 patient developed a desmoid type fibromatosis in the contralateral breast 14 months after breast-conserving surgery for breast cancer. In 1 patient, breast reduction surgery was performed on both breasts 11 years before the BDF operation, and 2 other patients underwent an excisional biopsy on the ipsilateral breast, with benign findings ( 2 and 8 years earlier). Overall, breast surgery was performed ipsilaterally in 5 out of 15 patients prior to the diagnosis of a desmoid type fibromatosis (Table 1).

\section{Clinical and Radiological Findings}

In 14 patients, the desmoid type fibromatosis was palpable. In 1 patient, a screening mammogram led to the detection of desmoid type fibromatosis, which was not detectable by palpation.

Desmoid type fibromatoses were palpable in 10 patients as a firm, partly severe parenchymal resistance. In 4 other patients, the tumor was palpable as a relatively smooth and sometimes movable nodule in the glandular tissue. In 4 patients, accompanying retraction was detectable (Table 1).

Mammography was performed in 13 patients. In 1 patient, on the basis of age (25 years), MRI of the breast was performed instead of mammography. In addition, mammography was omitted in the single man in the patient collective. In 1 patient, the BDF was not definable in mammography. In another patient, the only correlate was an architectural disorder of the breast parenchyma. As an irregularly bordered focus with additional spicules, BDF appeared in 5 patients (Fig. 1a). Another 5 patients showed a nonubiquitously smooth-edged lesion, and in 1 patient a circumscribed suspicious parenchymal density was detectable. Overall, mammography was classified as suspect in 12 patients with a Breast Imaging-Reporting and Data System (BI-RADS) score of 4 [19].

On ultrasound, BDF were found to be hypoechoic and irregular in all patients. Posterior acoustic shadowing was documented in 5 tumors, and speculated margins were documented in 2 tumors (Fig. 1b). In all ultrasound ex- 


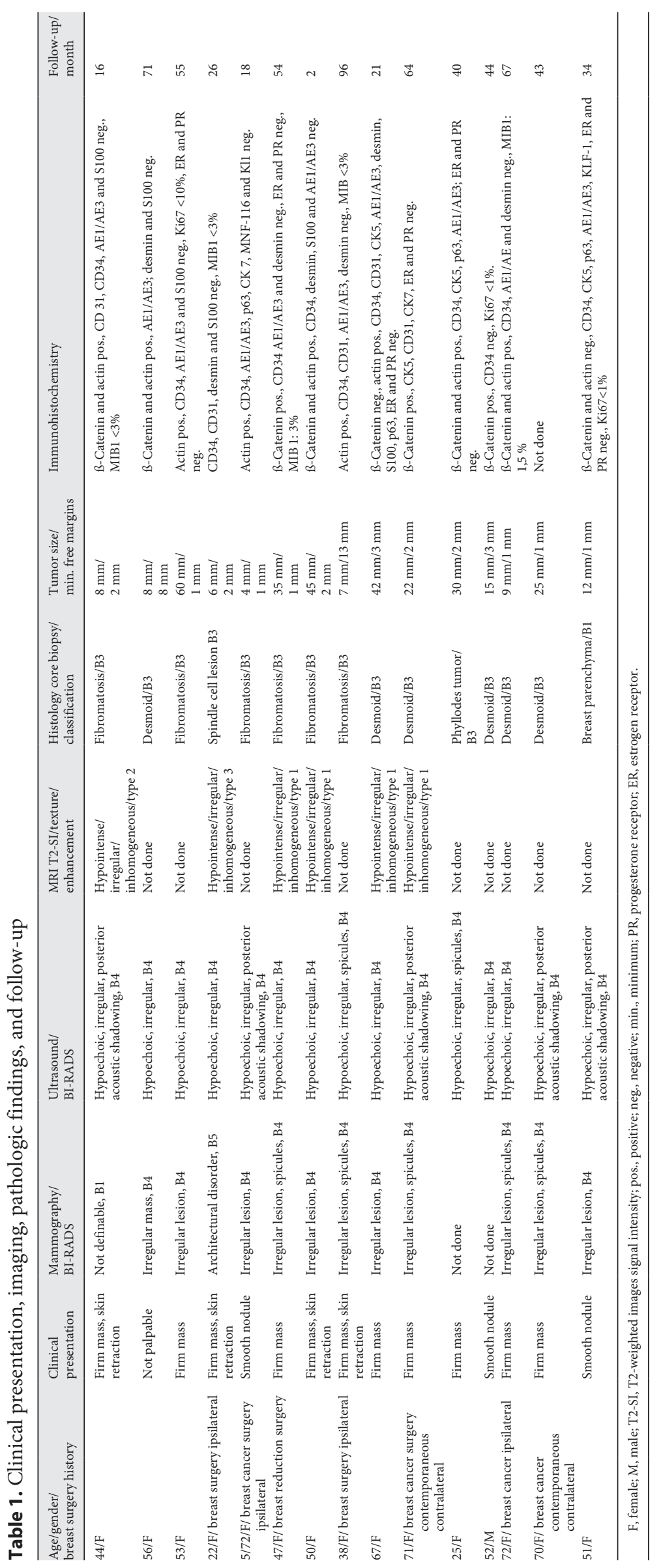


Fig. 1. a, b Seventy-two-year-old female patient with a palpable irregular mass in the right breast. A core biopsy histologically confirmed a desmoid type fibromatosis. a Mammography in craniocaudal projection with cutaneous marking of the palpation finding with a lead point: detection of a suspicious mass with an irregular border. b Ultrasound image: Hypoechoic irregular mass with posterior acoustic shadowing.

Fig. 2. a, b MRI of the breast of the same patient. a Spin echo T2-weighted image: hypointense irregular mass (arrow) without contact with the thoracic wall. b Subtracted T1-weighted postcontrast image: inhomogeneous, irregular contrast enhancement of the tumor.
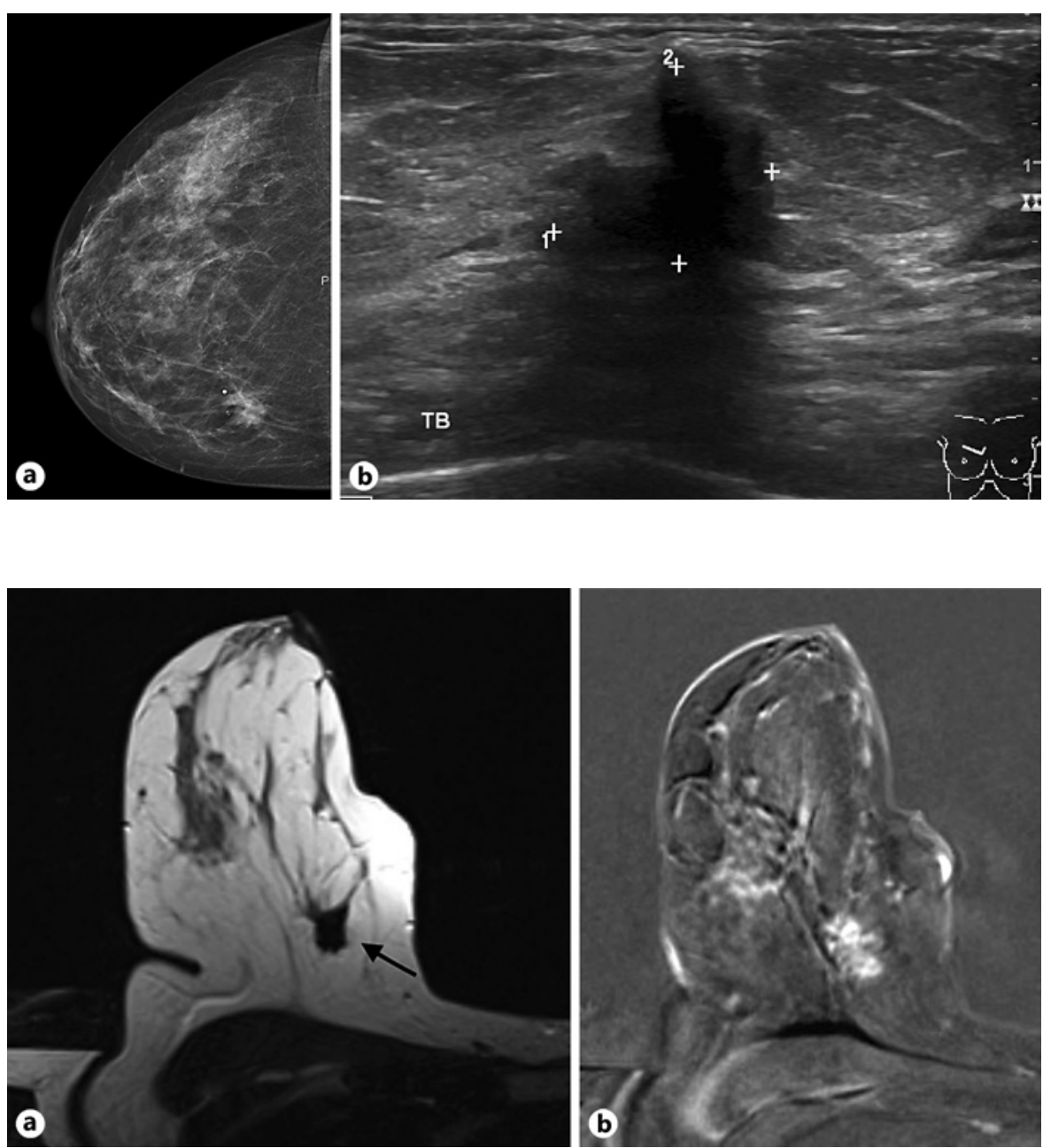

aminations, the tumor was classified as suspect in the assessment (BI-RADS 4) and histologically in need of clarification.

Six patients underwent preoperative MRI of the breast. In all patients BDF were hypointense on $\mathrm{T} 2$-weighted spin echo images and hypo- to isointense to the glandular tissue on T1-weighted images (Fig. 2a). Heterogeneous hyperintensity was documented in fat-saturated T2weighted images. In contrast, enhanced T1-weighted subtracted images demonstrated slow continuous contrast enhancement in 4 patients in the sense of benign type 1 kinetics [20]. The 2 remaining tumors showed a rather malignant-like rapid accumulation with a subsequent plateau (type 2 kinetics) or "wash-out" (type 3). In all 6 patients BDF was irregularly limited with inhomogeneous contrast enhancement and classified as suspicious in imaging (Fig. 2b).

\section{Preoperative and Postoperative Histology}

Preoperatively, an ultrasound-guided core biopsy was performed in all of the patients; an additional immunohistochemical examination of the specimen was per- formed in 11 patients. In 12 patients, the preoperative histological diagnosis of a desmoid type fibromatosis was made, and in 1 patient spindle cell proliferates were described, without malignancy-positive findings (category B3). The operative securing of the tumor was established according to the published guidelines [21]. In 1 patient, the external preoperative pathological examination of the core biopsy revealed the suspicion of a phyllodes tumor, but the patient did not undergo an additional immunohistochemical examination (category B3). In a further external pathological examination of another patient, only regular glandular tissue was found in the biopsy, without evidence of a tumor (Table 1).

For all surgical specimens whole-mount sections were prepared using standard stains (hematoxylin-eosin [HE], periodic acid-Schiff [PAS] reaction, and Masson-Goldner), and in 13 patients a supplementary immunohistochemical examination was performed. Only in the 70-year-old patient with concomitant contralateral extensive breast carcinoma was immunohistochemical examination of the core biopsy and the surgical specimen omitted. In this patient, the diagnosis 
Fig. 3. a, b Histological sections. a Wholemount sections in HE staining: The desmoid fibromatosis, which is shown centrally, infiltrates into the mammary gland tissue (arrow) with partly finger-like extensions. Top left: a higher magnification (13.3-fold) shows spindle cell proliferation without nuclear atypia. b Immunohistochemical staining with $\beta$-catenin at a 20 fold magnification: Predominantly nuclear staining of proliferating spindle cells is evident.

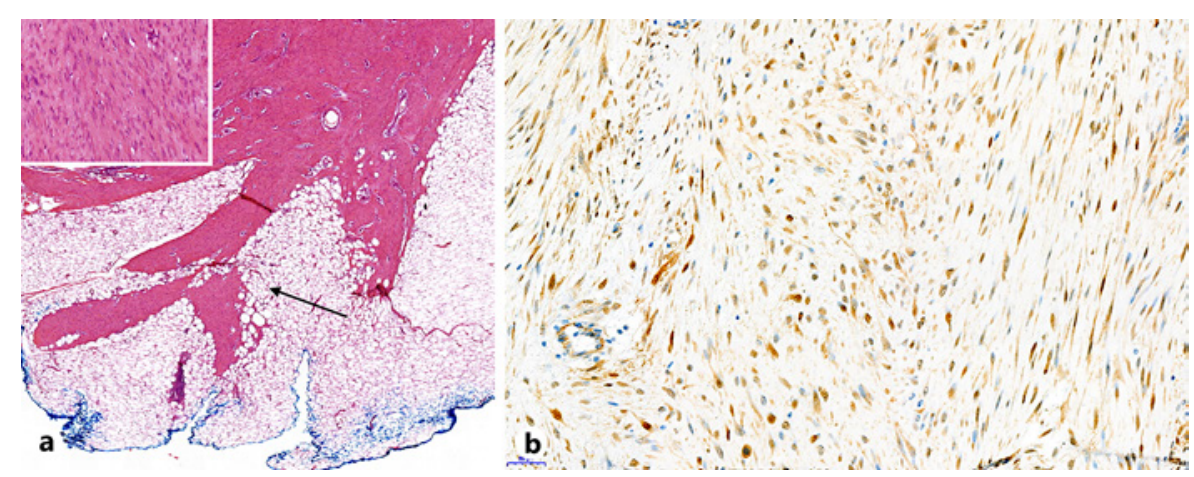

could be made on the basis of the standard stains described above (Fig. 3a).

For immunohistochemical studies the proto-oncoprotein marker $\beta$-catenin was used in 10 preparations, and in 8 patients the stain for $\beta$-catenin was positive (Fig. $3 \mathrm{~b}$ ). There were 6 cases with nuclear positivity and 1 case with cytoplasmic and nuclear staining. In 1 case only cytoplasmic positivity for $\beta$-catenin was detected. The smooth muscle marker actin was positive in 8 out of 10 specimens. Both patients with negative $\beta$-catenin had other complementary negative immunohistochemical staining which, in addition to microscopic histology, confirmed the diagnosis (e.g., negative for CD34, p63, CK5, and hormone receptors). The surface protein $\mathrm{CD} 34$ was negative in all 12 patients and CD31 was negative in all 5 patients examined. Antibodies against the pancytokeratins AE1/ AE3 were negative in all 11 patients examined (Table 1). The cytokeratins CK7 and CK5 were negative in all 5 cases. The proliferation marker Ki-67 or MIB- 1 was not increased in any of the 10 patients examined $(<10 \%)$. Other immunohistochemical assays for various antibodies, such as KL-1, MNF-116, p63, desmin, and S100, were negative in all of the patients examined. In addition, the hormone receptors (progesterone and estrogen) were studied in 5 patients, all of whom were negative.

\section{Treatment and Follow-Up}

All of the patients primarily underwent breast-conserving surgery with extended resection of the tumor area. An ultrasound hook wire localization of the findings was performed preoperatively in 2 patients, and in 5 patients an ultrasound verified skin marking of the tumor due to discrete palpation findings. There was complete resection of the tumor with free margins without the need for a second surgery. The smallest distance between BDF and healthy tissue averaged $2.8 \mathrm{~mm}$ (range: $1-13 \mathrm{~mm}$, median: $2 \mathrm{~mm}$ ). Further therapies, e.g., radiotherapy or systemic therapy, did not follow, in agreement with the postoperative tumor conference of the breast center.

The follow-up period with clinical and ultrasound controls in all of the patients and an additional mammog- raphy in 10 patients ranged from 16 to 96 months (mean: 44.86, median: 43 months). During this follow-up period, none of the patients was diagnosed with BDF recurrence. In 1 patient, breast cancer occurred 14 months after resection of the desmoid type fibromatosis in the contralateral breast.

\section{Discussion}

The genesis of desmoid type fibromatoses is unclear. However, their relationships with Gardner syndrome, previous trauma, and surgery have been described [1-5]. Furthermore, silicone implants are listed as a possible cause in the literature, although intraoperative trauma is more likely to be causative $[22,23]$. In the group investigated in this study, ipsilateral breast surgery was performed prior to the diagnosis of BDF in one third of the patients. Desmoid type fibromatoses typically occur between the 15th and 60th years of life [1-5]. The age of diagnosis was also broad in this study, with an age range of 22-72 years. In women, desmoid type fibromatoses are diagnosed twice as often as in men, so a hormonal influence is also discussed $[1,2,5,14,24]$.

Clinically, this tumor manifests as a broad spectrum. In most cases, desmoid type fibromatosis of the breast is described as a suspicious, mobile, firm, and painless nodule. However, cutaneous skin retractions and nipple retractions have also been reported in the literature $[3,5-7$, $15,16]$. In this study, BDF had a palpatory, predominantly also suspicious and firm consistency. In 4 patients, skin retraction was delimited.

On imaging, BDF in mammography presented in this study as irregularly limited malignancy-related masses, partly with spicules and without evidence of microcalcifications. Also in agreement with the previous literature descriptions, sonographically, predominantly hypoechoic and irregular lesions were found in the examined group, partly with concomitant dorsal sound cancellation [2528]. All of the tumors met the imaging criteria for a malignancy-related finding. 
MRI of BDF is used to estimate the overall extent and exclusion of thoracic wall infiltrations; postoperatively, it serves to eliminate any possible residual tumor components. The literature on the MRI of desmoid type fibromatosis of the breast consists predominantly of individual case descriptions $[24,29,30]$.

The preoperative MR mammograms of the tumors in the present study showed an inconsistent image of contrast medium enhancement. Thus, both slow and continuous enhancement patterns as well as rapid enhancement with a subsequent plateau or "wash-out" were observed. This heterogeneous and nonspecific contrast kinetics is attributed to the varying proportion of celland collagen-rich tissue $[29,30]$.

In this cohort, preoperative core biopsy examinations were carried out on all patients, which revealed the suspicion of fibromatosis or a desmoid type fibromatosis in $86 \%$ of the tissue samples obtained. A malignancyrelated finding was not properly diagnosed. An independent factor in event-free survival of desmoid type fibromatosis is the way the diagnostic biopsy is obtained, with a clear advantage of core biopsy over open biopsy [12]. Based on this data, the importance of a careful preoperative core biopsy securing the diagnosis of a desmoid type fibromatosis becomes clear once again. According to the literature, partly documented axillary dissections and unnecessary secondary operations can be avoided $[5,31]$.

Histological diagnosis of BDF is based on the morphological characteristics of fibromatosis and can be diagnosed by common histological staining [31-36]. In 1 case in our study, the diagnosis was also made only on the surgical specimen by macroscopic and microscopic imaging. Overall, however, due to the variable histological presentation of desmoid type fibromatosis, primary false diagnoses occur in up to $58 \%$ of cases [37].

Immunohistochemical analysis, especially of preoperative biopsies, can facilitate the diagnosis $[31,33]$. In $70 \%$ of the BDF in this study nuclear $\beta$-catenin staining was detected. In addition, the immune marker actin was positive in smooth muscle cells in $80 \%$ of the preparations. $\beta$-Catenin is a key immunohistochemical indicator for a desmoid fibromatosis and shows nuclear expression in sporadic and familial cases in up to $80 \%$ of patients [ 31 , $33,38]$. Mutations in the $\beta$-catenin gene (CTNNB1) are found in most sporadic desmoid type fibromatosis. These genetic changes lead to the accumulation of this oncoprotein in the nucleus $[38,39]$.

Elevated $\beta$-catenin levels stimulate the WnT signaling pathway, which is known to play a role in tumor genesis $[39,40]$. Nevertheless, nuclear $\beta$-catenin staining is not specific for BDF and it is observed rarely in spindle cell carcinomas, $23 \%$ of metaplastic breast carcinomas, and up to $93 \%$ of benign phyllodes tumors [38].
In addition, negative staining for CD34 was observed in all of the patients examined in the current study. CD34 negativity may be a further indication of breast fibromatosis. It is described only in nodular fasciitis for stromal breast lesions and fibromatosis [31, 35, 41]. A low Ki-67 index and negativity for pan-cytokeratin (e.g., AE1/AE3), cytokeratin (e.g., CK5 and CK7) and hormone receptors, which were observed in all of the patients investigated in this study, are further pathological criteria for mammary desmoid type fibromatosis [31, 33, 35, 41].

A primary nonsurgical approach with active monitoring of the patient is recommended in ESMO guidelines since 2017, as evaluations showed no progress or regression in desmoid patients in up to $88 \%$ of cases $[8,9,11$, 12]. The prerequisite is the unambiguous core biopsy assurance of the findings and close follow-up controls by MRI examinations [8-12]. Overall, an active surveillance strategy is preferred in desmoid type fibromatosis with unfavorable locations, which do not include the breast [12].

Nevertheless, the data to date are classified as "very low" according to GRADE (Grading of Recommendations Assessment, Development and Evaluation) by the Desmoid Tumor Working Group [9]. In the case of required active treatment, surgery is still considered as the first-line therapy under the condition of low expected morbidity [8-12].

In the rare case of a desmoid type fibromatosis of the breast, a recent first large cohort study from Duazo-Cassin et al. [11] showed that 15 out of 17 patients with primary active monitoring did not require further therapy. On the other hand, the group of 46 patients undergoing primary surgery in this study showed only a low recurrence rate of $8.7 \%$ (4 patients). Notably, there was no relapse in our study population over a median follow-up interval of 43 months. A possible explanation for this result is that all 15 patients with negative margins underwent primary wide excision of the tumor and did not therefore need further surgery, with otherwise comparable tumor sizes and patient ages that were otherwise comparable to those in the study of Duazo-Cassin et al. [11].

Data on the importance of tumor-free margins are controversial and at least in the overall group of desmoid type fibromatosis free surgical margins do not seem to be a constant risk factor for recurrence [5, 8-14]. In a recent meta-analysis of 16 studies with a total of 1,295 patients with extra-abdominal manifestations of desmoid type fibromatosis, the risk of recurrence was again almost twice as high with positive surgical margins. However, the different tumor locations were not explicitly differentiated in this study and desmoid type fibromatosis of the breast made up only a small part of the cases examined [42].

Further therapies did not follow, in agreement with the postoperative tumor conference in this study. The use of
Lorenzen/Cramer/Buck/Friedrichs/ Graubner/Lühr/Lindner/Niendorf 
adjuvant radiotherapy remains controversial without a significant benefit and it is therefore not recommended, especially in young patients with the risk of late morbidity $[4,8,9]$. Radiation therapy is only considered in the case of a recurrence of desmoid type fibromatosis, or in cases of inoperable recurrence, with the need for active treatment $[8,9]$.

For systemic therapy, approaches with tamoxifen or nonsteroidal anti-inflammatory drugs, sometimes in combination, with different response rates to complete remission are described without the mechanism of action being completely clear $[4,43,44]$. Likewise, there are approaches with tyrosine kinase inhibitors in progressive desmoid type fibromatosis and relapses with partial and complete remission $[44,45]$. However, there is a general lack of randomized studies on the use of medical treatment for desmoid type fibromatosis. Therefore, in aggressive cases an adapted therapy is currently recommended, taking into account the toxic side effects $[4,8$, 9]. This should be applied depending on the growth rate of the tumor and the urgency of the therapy $[8,9]$.

\section{Conclusion}

Clinically, especially on imaging, desmoid type fibromatosis has typical malignant criteria. In the majority of patients, however, a preoperative core biopsy can be used to prove a desmoid type fibromatosis histologically and, for instance, with additional positive evidence of $\beta$-catenin and negativity for CD34, pan- and basal cytokeratin, and p63 in immunohistochemistry. In the case of an active treatment of BDF by surgical intervention, detailed preoperative imaging and histological diagnosis then allow planning of a complete primary excision of fibromatosis with free resection margins and a reduced risk of recurrence.

\section{Statement of Ethics}

This study complies with the guidelines for human studies regulations. All of the patients gave their written informed consent for evaluation of their anonymized data for this study according to the requirements and with institutional board approval of the local ethics committee (Medical Association Hamburg; WF-22/20).

\section{Disclosure Statement}

The authors declare no conflict of interests.

\section{Funding Sources}

None.

\section{Author Contributions}

J.L.: contribution to development of the study design, collection of data, analysis and interpretation of data, statistical analysis, and drafting this paper. M.C.: collection of data, interpretation of data, and drafting of this paper. N.B.: contribution to development of the study design, collection of data, and drafting of this paper. K.F.: analysis and interpretation of data, contribution to implementation in one of the participating institutes, and drafting of this paper. K.B.: collection of data, contribution to implementation in one of the participating institutes, and drafting of this paper. C.S.L.: attendance and evaluation in selection of image data sets, collection of data, and drafting of this paper. C.L.: drafting and revision of this paper, contribution to implementation in one of the participating institutes, and conception of this study. A.N.: contribution to implementation in one of the participating institutes, conception and design of this study, and drafting and revision of this paper.

All of the authors read and approved the final version of this paper to be published and have confidence in the integrity of the contributions of their coauthors. All of the authors are accountable for all aspects of this work related to the accuracy or integrity of any part of this study.

\section{References}

1 Posner MC, Shiu MH, Newsome JL, Hajdu SI, Gaynor JJ, Brennan MF. The desmoid tumor. Not a benign disease. Arch Surg. 1989 Feb; 124(2):191-6.

2 Sakorafas GH, Nissotakis C, Peros G. Abdominal desmoid tumors. Surg Oncol. 2007 Aug;16(2):131-42.

3 Rosen PP, Ernsberger D. Mammary fibromatosis. A benign spindle-cell tumor with significant risk for local recurrence. Cancer. 1989 Apr;63(7):1363-9.

4 Eastley N, McCulloch T, Esler C, Hennig I, Fairbairn J, Gronchi A, et al. Extra-abdominal desmoid fibromatosis: A review of management, current guidance and unanswered questions. Eur J Surg Oncol. 2016 Jul;42(7):1071-83.

5 Neuman HB, Brogi E, Ebrahim A, Brennan MF, Van Zee KJ. Desmoid tumors (fibromatoses) of the breast: a 25-year experience. Ann Surg Oncol. 2008 Jan;15(1):274-80.
6 Grimaldi MC, Trentin C, Lo Gullo R, Cassano E. Fibromatosis of the breast mimicking cancer: A case report. Radiol Case Rep. 2017 Nov; 13(1):1-5.

7 Cederlund CG, Gustavsson S, Linell F, Moquist-Olsson I, Andersson I. Fibromatosis of the breast mimicking carcinoma at mammography. Br J Radiol. 1984 Jan;57(673):98101.

8 Kasper B, Baumgarten C, Garcia J, Bonvalot $S$, Haas R, Haller F, et al.; Desmoid Working Group. An update on the management of sporadic desmoid-type fibromatosis: a European Consensus Initiative between Sarcoma PAtients EuroNet (SPAEN) and European Organization for Research and Treatment of Cancer (EORTC)/Soft Tissue and Bone Sarcoma Group (STBSG). Ann Oncol. 2017 Oct; 28(10):2399-408.
9 Desmoid Tumor Working Group: The management of desmoid tumours: a joint global consensus based guideline approach for adult and paediatric patients. Eur J Cancer. 2020; 27:96-107.

10 Roussin S, Mazouni C, Rimareix F, Honore C, Terrier P, Mir O, et al. Toward a new strategy in desmoid of the breast? Eur J Surg Oncol. 2015 Apr;41(4):571-6.

11 Duazo-Cassin L, Le Guellec S, Lusque A, Chantalat E, Lae M, Terrier P, et al. Breast desmoid tumor management in France: toward a new strategy. Breast Cancer Res Treat. 2019 Jul;176(2):329-35.

12 Penel N, Le Cesne A, Bonvalot S, Giraud A, Bompas E, Rios M, et al. Surgical versus nonsurgical approach in primary desmoid-type fibromatosis patients: a nationwide prospective cohort from the French Sarcoma Group. Eur J Cancer. 2017 Sep;83:125-31. 
13 Ballo MT, Zagars GK, Pollack A, Pisters PW, Pollack RA. Desmoid tumors: prognostic factors and outcome after surgery and radiation therapy. J Clin Oncol. 1999;17:158-67.

14 Gump FE, Sternschein MJ, Wolff M. Fibromatosis of the breast. Surg Gynecol Obstet. 1981 Jul;153(1):57-60.

15 Wargotz ES, Norris HJ, Austin RM, Enzinger FM. Fibromatosis of the breast. A clinical and pathological study of 28 cases. Am J Surg Pathol. 1987 Jan;11(1):38-45.

16 Erguvan-Dogan B, Dempsey PJ, Ayyar G, Gilcrease MZ. Primary desmoid tumor (extraabdominal fibromatosis) of the breast. AJR Am J Roentgenol. 2005 Aug;185(2):488-9.

17 Glazebrook KN, Reynolds CA. Mammary fibromatosis. AJR Am J Roentgenol. 2009 Sep; 193(3):856-60.

18 Ebrahim L, Parry J, Taylor DB. Fibromatosis of the breast: a pictorial review of the imaging and histopathology findings. Clin Radiol. 2014 Oct;69(10):1077-83.

19 D'Orsi CJ, Sickles EA, Mendelson EB, Morris EA, et al. ACR BI-RADS(R) Atlas, Breast Imaging Reporting and Data System. Reston: American College of Radiology; 2013.

20 Kuhl CK, Mielcareck P, Klaschik S, Leutner C, Wardelmann E, Gieseke J, et al. Dynamic breast MR imaging: are signal intensity time course data useful for differential diagnosis of enhancing lesions? Radiology. 1999 Apr; 211(1):101-10.

21 Amendoeira I. Quality assurance guidelines for pathology: cytological and histological non-operative procedures. In: Perry NM (editor). European guidelines for quality assurance in breast cancer screening and diagnosis. Office for Official Publications of the European Communities, 2006. p. 221-56.

22 Tzur R, Silberstein E, Krieger Y, Shoham Y, Rafaeli Y, Bogdanov-Berezovsky A. Desmoid tumor and silicone breast implant surgery: is there really a connection? A literature review. Aesthetic Plast Surg. 2018 Feb;42(1):59-63.

23 Vandeweyer E, Deraemaecker R. Desmoid tumor of the breast after reconstruction with implant. Plast Reconstr Surg. 2000 Jun; 105(7):2627-8.

24 Plaza MJ, Yepes M. Breast fibromatosis response to tamoxifen: dynamic MRI findings and review of the current treatment options. J Radiol Case Rep. 2012 Mar;6(3):16-23.
25 Samardzic T, Lomo J, Skaane P. Screeningdetected desmoid tumor of the breast: findings at conventional imaging and digital breast tomosynthesis. Acta Radiol Open. 2018 Jan;7(1):2058460117752034.

26 Leibman AJ, Kossoff MB. Sonographic features of fibromatosis of the breast. J Ultrasound Med. 1991 Jan;10(1):43-5.

27 Lopez-Ruiz J, Ruiz M, Echevarria JJ, Lopez S, Imaz I. Mammary fibromatosis mimicking recurrent breast cancer: radiological findings. Eur Radiol. 2005 Sep;15(9):2034-6.

28 Alanis L, Roth R, Lerman N, Barroeta JE, Germaine P. Radiologic images of an aggressive implant-associated fibromatosis of the breast and chest wall: case report and review of the literature. Radiol Case Rep. 2017 Jun;12(3): 431-8.

29 Nakazono T, Satoh T, Hamamoto T, Kudo S. Dynamic MRI of fibromatosis of the breast. AJR Am J Roentgenol. 2003 Dec;181(6): 1718-9.

30 Mesurolle B, Leconte I, Fellah L, Feger C, Nakazono T, Kudo S. Dynamic breast MRI in recurrent fibromatosis. AJR Am J Roentgenol. 2005 Feb;184(2):696-7.

31 Kuba MG, Lester SC, Giess CS, Bertagnolli MM, Wieczorek TJ, Brock JE. Fibromatosis of the Breast: Diagnostic Accuracy of Core Needle Biopsy. Am J Clin Pathol. 2017 Sep;148(3): 243-50.

32 Devouassoux-Shisheboran M, Schammel MD, Man YG, Tavassoli FA. Fibromatosis of the breast: age-correlated morphofunctional features of 33 cases. Arch Pathol Lab Med. 2000 Feb;124(2):276-80.

33 Magro G, Salvatorelli L, Puzzo L, Piombino E, Bartoloni G, Broggi G, et al. Practical approach to diagnosis of bland-looking spindle cell lesions of the breast. Pathologica. 2019 Dec;111(4):344-60.

34 Tse GM, Tan PH, Lui PC, Putti TC. Spindle cell lesions of the breast-the pathologic differential diagnosis. Breast Cancer Res Treat. 2008 May;109(2):199-207.

35 Lee AH. Recent developments in the histological diagnosis of spindle cell carcinoma, fibromatosis and phyllodes tumour of the breast. Histopathology. 2008 Jan;52(1):45-57.
36 Schnitt S, Collins LC. Biopsy interpretation of the breast. 3rd ed. Philadelphia: Lippincott Williams; 2013

37 Huss S, Nehles J, Binot E, Wardelmann E, Mittler J, Kleine MA, et al. $\beta$-catenin (CTNNB1) mutations and clinicopathological features of mesenteric desmoid-type fibromatosis. Histopathology. 2013 Jan;62(2):294-304.

38 Lacroix-Triki M, Geyer FC, Lambros MB, Savage K, Ellis IO, Lee AH, et al. $\beta$-catenin/ Wnt signalling pathway in fibromatosis, metaplastic carcinomas and phyllodes tumours of the breast. Mod Pathol. 2010 Nov; 23(11):1438-48.

39 Crago AM, Chmielecki J, Rosenberg $M$ O'Connor R, Byrne C, Wilder FG, et al. Near universal detection of alterations in CTNNB1 and Wnt pathway regulators in desmoid-type fibromatosis by whole-exome sequencing and genomic analysis. Genes Chromosomes Cancer. 2015 Oct;54(10):606-15.

40 Bienz M, Clevers H. Linking colorectal cancer to Wnt signaling. Cell. 2000 Oct;103(2):31120.

41 Rakha EA, Aleskandarany MA, Lee AH, Ellis IO. An approach to the diagnosis of spindle cell lesions of the breast. Histopathology. 2016 Jan;68(1):33-44.

42 Janssen ML, van Broekhoven DL, Cates JM, Bramer WM, Nuyttens JJ, Gronchi A, et al. Meta-analysis of the influence of surgical margin and adjuvant radiotherapy on local recurrence after resection of sporadic desmoid-type fibromatosis. Br J Surg. 2017 Mar; 104(4):347-57.

43 Janinis J, Patriki M, Vini L, Aravantinos G, Whelan JS. The pharmacological treatment of aggressive fibromatosis: a systematic review. Ann Oncol. 2003 Feb;14(2):181-90.

44 Scheer L, Lodi M, Molière S, Kurtz JE, Mathelin C. Medical treatment of mammary desmoid-type fibromatosis: which benefit? World J Surg Oncol. 2017 Apr;15(1):86.

45 Penel N, Le Cesne A, Bui BN, Perol D, Brain EG, Ray-Coquard I, et al. Imatinib for progressive and recurrent aggressive fibromatosis (desmoid tumors): an FNCLCC/French Sarcoma Group phase II trial with a longterm follow-up. Ann Oncol. 2011 Feb;22(2): 452-7. 